ARTICLE

https://doi.org/10.1038/s41467-019-13649-6

\title{
A bacterial gene-drive system efficiently edits and inactivates a high copy number antibiotic resistance locus
}

\author{
J. Andrés Valderrama ${ }^{1,2}$, Surashree S. Kulkarni ${ }^{1,3}$, Victor Nizet (iD ${ }^{1,2,4 \star}$ \& Ethan Bier ${ }^{1,3 \star}$
}

Gene-drive systems in diploid organisms bias the inheritance of one allele over another. CRISPR-based gene-drive expresses a guide RNA (gRNA) into the genome at the site where the gRNA directs Cas9-mediated cleavage. In the presence of Cas9, the gRNA cassette and any linked cargo sequences are copied via homology-directed repair (HDR) onto the homologous chromosome. Here, we develop an analogous CRISPR-based gene-drive system for the bacterium Escherichia coli that efficiently copies a gRNA cassette and adjacent cargo flanked with sequences homologous to the targeted gRNA/Cas9 cleavage site. This "proactive" genetic system (Pro-AG) functionally inactivates an antibiotic resistance marker on a high copy number plasmid with 100-fold greater efficiency than control CRISPR-based methods, suggesting an amplifying positive feedback loop due to increasing gRNA dosage. Pro-AG can likewise effectively edit large plasmids or single-copy genomic targets or introduce functional genes, foreshadowing potential applications to biotechnology or biomedicine.

\footnotetext{
${ }^{1}$ Tata Institute for Genetics and Society, University of California, San Diego, 9500 Gilman Drive, La Jolla, CA 92093-0349, USA. ${ }^{2}$ Collaborative to Halt Antibiotic-Resistant Microbes, Department of Pediatrics, University of California, San Diego, 9500 Gilman Drive, La Jolla, CA 92093-0760, USA. ${ }^{3}$ Section of Cell and Developmental Biology, University of California, San Diego, 9500 Gilman Drive, La Jolla, CA 92093-0349, USA. ${ }^{4}$ Skaggs School of Pharmacy \& Pharmaceutical Sciences, University of California, San Diego, 9500 Gilman Drive, La Jolla, CA 92093-0760, USA. *email: vnizet@ucsd.edu; ebier@ucsd.edu
} 
S ynthetic gene-drive elements based on bacterial-derived CRISPR components have been developed in diploid organisms including yeast ${ }^{1}$, insects ${ }^{2-4}$, and mammals ${ }^{5}$. The salient feature of these "active genetic" systems is that a guide RNA (gRNA) is directly flanked by sequences homologous to the genomic site it targets for Cas9-mediated cleavage. When doublestranded DNA breaks are induced in the germline by the gRNA/ Cas9 complex, the gRNA together with any linked cargo sequences are then copied into the break via the homologydirected repair (HDR) pathway ${ }^{6}$. Such gene conversion events can greatly bias transmission of the gRNA cassette so that it is inherited by nearly all progeny.

Echoing their functional CRISPR origins, synthetic bipartite gRNA/Cas9 systems have been developed in bacteria ${ }^{7-9}$, including targeting of plasmid-encoded antibiotic resistance determinants ${ }^{10}$. Indeed, as plasmid-borne antibiotic resistance genes and virulence determinants are important in the pathogenesis of many human bacterial infections, inactivation or replacement of such multi-copy targets could greatly impact the success or failure of treatment interventions ${ }^{11,12}$. We contemplated whether modifying a standard bacterial CRISPR system by developing an active self-copying mechanism could enhance efforts to scrub antibiotic resistance in prokaryotes ("pro-active genetics" or ProAG) and thereby address the challenge of high gene dosage on multi-copy plasmids.

Here, we develop a Pro-AG gene-drive system for the bacterium Escherichia coli that efficiently copies a functional gRNA cassette flanked with sequences homologous to the targeted gRNA/Cas9 cleavage site. Pro-AG inactivates an antibiotic resistance marker on a high copy number plasmid with $\sim 100$-fold greater efficiency than control CRISPR-based methods, indicating a self-amplifying positive feedback loop linked to increasing gRNA dosage. Likewise, the system can efficiently edit a large plasmid, target genes on the bacterial chromosome, or be adapted to introduce functional gene cargos alongside the gRNA cassette. Pro-AG expands the available toolkit for engineering or manipulating bacteria in future biotechnology and biomedicine applications.

\section{Results}

Establishment of the pro-active genetics (Pro-AG) system. In our first set of experiments, we employed a set of three mutually compatible plasmids to assess Cas9-mediated cleavage/inactivation of the beta-lactamase (bla) target gene, which confers ampicillin resistance $\left(\mathrm{Amp}^{\mathrm{R}}\right)$ in Escherichia coli. The three plasmids were: (1) high copy number pET bearing the bla target gene and conferring Amp ${ }^{\mathrm{R}}$; (2) low copy pCRISPR Amp carrying one of two different gRNAs targeting bla sequences under constitutive transcriptional control and maintained under spectinomycin selection $\left(\mathrm{Spm}^{\mathrm{R}}\right)$; and (3) low copy pCas9-expressing Cas9 under control of a tet promoter, inducible with anhydrotetracycline (aTc) and propagated under chloramphenicol selection $\left(\mathrm{Cm}^{\mathrm{R}}\right)$ (Fig. 1a; Supplementary Table 1).

Initial transformation of $E$. coli with the compatible threeplasmid system (scheme in Supplementary Fig. 1a) did not lead to significant differences in colony-forming unit (CFU) recovery on triple antibiotic $(\mathrm{Amp}+\mathrm{Spm}+\mathrm{Cm})$ agar plates either in the presence $(+\mathrm{aTc})$ or absence $(-\mathrm{aTc})$ of anhydrotetracycline induction with either of two different gRNAs targeting bla sequences (Supplementary Fig. 2a). Individual E. coli colonies carrying all three plasmids were then tested under an overnight growth protocol that imposes multi-generational antibiotic selection (see Supplementary Fig. 1 and Methods). In brief, colonies inoculated in Luria broth (LB) were grown overnight, maintaining antibiotic selection with or without aTc induction
(Supplementary Fig. 1a). Although terminal culture densities were unaltered by Cas9 induction (Supplementary Fig. 2b), culture aliquots regrown under the more-stringent conditions imposed by selection on triple-antibiotic plates displayed significant Cas9-dependent reductions in CFU for both gRNA1 $(\sim 10$-fold) and gRNA2 ( 100-fold) (Fig. 1b). These differential responses to the Cas9 induction regimen are likely attributable to non-autonomous antibiotic rescue of mixed cells by secreted Bla in overnight liquid culture, absent during antibiotic plate selection of well isolated colonies. This strategy using CRISPRCas9 alone (e.g., without a homology template) is referred to hereafter as CRISPR-control.

As the targeting efficiency of gRNA2 under the aforementioned CRISPR-control framework matched previously reported reductions in CFU using a similar experimental paradigm ${ }^{10}$, we chose to test this gRNA in a "pro-active" configuration (Pro-AG) (Fig. 1c). We modified the $\mathrm{Spm}^{\mathrm{R}}$ gRNA-expressing plasmid by flanking the gRNA2 expression cassette with bla sequence homology arms (HA, $500 \mathrm{bp}$ each, comparable in length to HA employed in eukaryotic systems) that directly abut the gRNA cleavage site, generating the pPro-AG plasmid (Supplementary Table 1). Since the gRNA cleavage site is absent from this plasmid, the encoded gRNA is unable to cleave these sequences in the presence of Cas9. Because standard E. coli strains do not support efficient homology-based insertion of genomic cassettes following induction of double-strand DNA breaks ${ }^{13,14}$, we made use of a cassette encoding recombinogenic lambda-Red $(\lambda \text { Red })^{15,16}$ enzymes under control of an arabinose inducible promoter (arab) that is also encoded on this plasmid ${ }^{9}$. Plasmids pET (carrying the bla $\mathrm{Amp}^{\mathrm{R}}$ target gene) and pCas9 were identical to those used for the "CRISPR- control" regimen (Fig. 1c). Following the parallel experimental scheme depicted in Supplementary Fig. 1b, we again observed no significant differences in CFU following initial transformation of the three Pro-AG plasmid components under triple-antibiotic (Amp + $\mathrm{Spm}+\mathrm{Cm}$ ) selection with or without Cas9 induction (i.e., $\pm \mathrm{aTc}$ ) (Supplementary Fig. 3a). Similarly, Cas $9 \pm \lambda$ Red induction with aTc or arabinose, respectively, did not appreciably impact overnight culture densities under triple-antibiotic selection (Supplementary Fig. 3b).

Pro-AG efficiently inactivates antibiotic resistance. As an initial comparison between the CRISPR-control (Fig. 1a) and the ProAG (Fig. 1c) configurations, overnight cultures of E. coli colonies transformed with the corresponding three-plasmid systems were grown in triple antibiotics with or without aTc (to induce Cas9), alone or in combination with arabinose (to induce $\lambda$ Red enzymes), then plated for CFU enumeration (Fig. 1d). In contrast to $\sim 100$-fold CFU reduction observed with the gRNA using our "CRISPR-control" component configuration (Fig. 1b, d), targeting of bla using the Pro-AG format (i.e., induction of Cas9 $+\lambda$ Red) led to a $\sim 100,000$-fold reduction in $\mathrm{Amp}^{\mathrm{R}} \mathrm{CFU}$ (Fig. 1d). For reasons that remain unclear, induction of $\lambda$ Red also yielded a modest reduction in CFU recovery in the CRISPR-control regimen; however, this effect was much more pronounced in a Pro-AG context (Fig. 1d, green vs. blue shading). The intensified reduction in $\mathrm{Amp}^{\mathrm{R}}$ E. coli CFU could not be attributed to secondary effects of aTc and/or Cas9 induction, since no significant differences in CFU were observed under Amp selection (+ Amp) without Cas9 induction $(-\mathrm{aTc})$ compared with Cas9 induction $(+\mathrm{aTc})$ without Amp selection (-Amp) (Supplementary Fig. 4a). Additional controls excluded suppressive effects of arabinose or $\lambda$ Red enzyme expression per se, as arabinose induction alone did not alter CFU recovery (Supplementary Fig. 4b). 
a

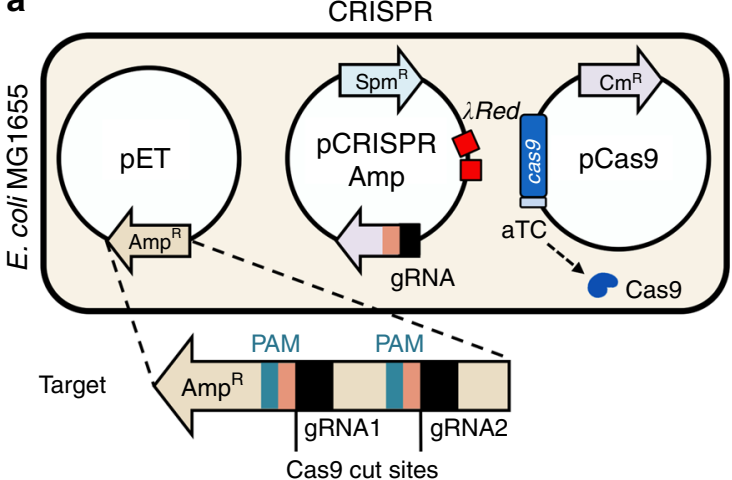

C

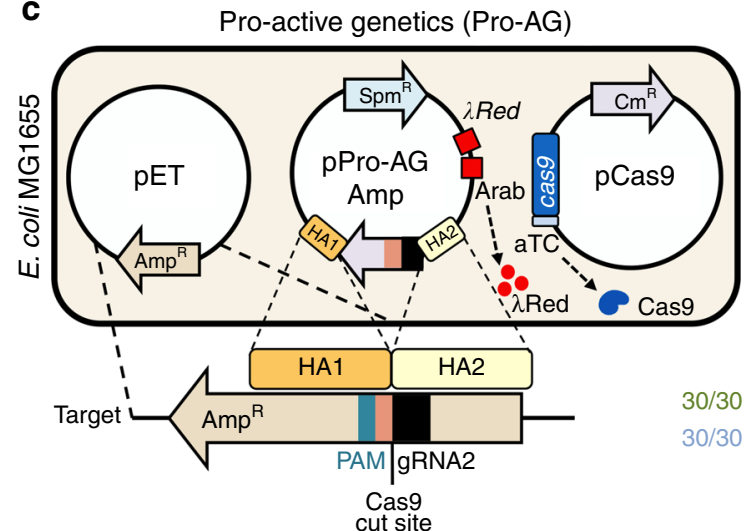

b
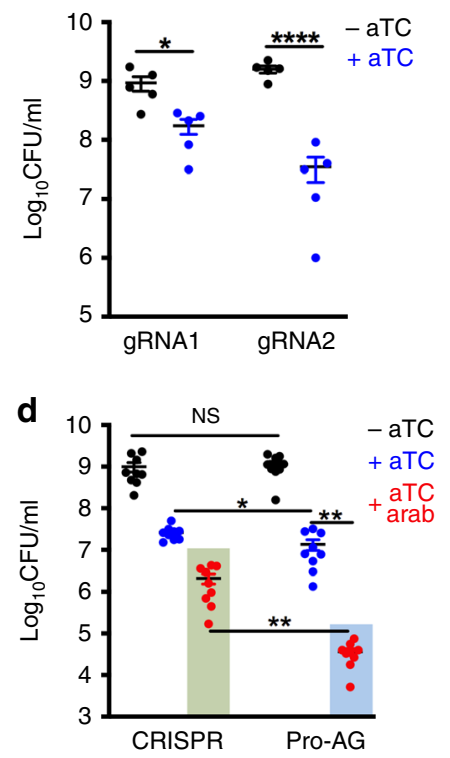

$\mathrm{Amp}^{\mathrm{R}}$ escapers analysis PAM AmpgRNA2

CCG CTG TTGAGATCCAGTTCGAT

3' CCG CTG TTGAGATCCAGTTCGAT 5'

Cas9

cut site

Fig. 1 Pro-active genetics (Pro-AG) is 100x more efficient than CRISPR-control for targeting antibiotic resistance conferred by a high copy plasmid. $\mathbf{a}$ and $\mathbf{c}$ Schematic of CRISPR-control- a and Pro-AG- c mediated editing of beta-lactamase gene (bla) encoded on a high copy plasmid ( $p E T$ ) conferring resistance to ampicillin $\left(A \mathrm{mp}^{\mathrm{R}}\right.$, tan arrow) in E. coli MG1655. gRNAs were initially expressed from a low copy plasmid (pCRISPR Amp) maintained under spectinomycin ( $\mathrm{Spm}$ ) selection and using the constitutive tet promoter to express the gRNA. A second low copy plasmid, pCas9, maintained under chloramphenicol $\left(\mathrm{Cm}^{\mathrm{R}}\right)$ selection, encodes Cas9 under control of an anhydrotetracycline (aTc) inducible promoter. a CRISPR-control configuration: two gRNAs (gRNA1 and gRNA2 carried on the pCRISPR Amp plasmid) were tested for Cas9-mediated targeting at two different locations in the bla gene. The Cas9/gRNA1 and Cas9/gRNA2-induced cleavage sites, and the protospacer adjacent motif (PAM) are indicated as well as a gene cassette carrying the $\lambda$ Red enzymes. b Recovery of $A \mathrm{mp}^{\mathrm{R}}$ colony-forming units ( $\mathrm{Log}_{10} \mathrm{CFU} / \mathrm{ml}$ ) following CRISPR-mediated targeting of the bla gene with gRNAs 1 or 2 in the absence ( $-\mathrm{aTc}$, black dots) or presence ( + aTc, blue dots) of aTc-induced Cas9 expression. c Pro-AG configuration: the gRNA2 expression cassette flanked by bla $\left(\mathrm{Amp}^{R}\right)$ homology arms (HA1 and HA2) that directly abut the gRNA2 cleavage site was incorporated into pCRISPR Amp. The Cas9/gRNA2induced cleavage site, the protospacer adjacent motif (PAM), and the HA1 (dark yellow box), and HA2 (light yellow box) homology arms are indicated. Also carried on plasmids pCRISPR Amp and pPro-AGAmp are the recombinogenic $\lambda$ Red enzymes ( $\lambda$ Red), which can be induced with L-arabinose (arab). d Recovery of CFU on Amp plates following CRISPR-control versus Pro-AG-mediated targeting of the bla gene. In this and subsequent panels, abbreviations for Cas9 induction are as in panel $\mathbf{b}$; induction of $\lambda$ Red enzymes: ( + aTc + arab: red dots); CRISPR (green shaded box) and Pro-AG (blue shaded box) treatments are highlighted. Plasmids sequenced from Amp ${ }^{R}$ colonies (CRISPR, green box and Pro-AG, blue box) displayed unaltered gRNA target sites in all clones analyzed (30/30). Data in $\mathbf{b}$ and $\mathbf{d}$ are plotted as the mean $\pm S E M$, representing three independent experiments performed in triplicate and analyzed by Student's $t$ test. N.S. $=$ not significant $(P>0.05){ }^{\star} P<0.05 ;{ }^{\star \star} P<0.01 ;{ }^{\star \star \star \star} P<0.0001$. Source Data are available in the Source Data file.

We expected that either unrepaired CRISPR-mediated doublestranded DNA breaks or potential Pro-AG-mediated insertion of gRNA2 sequences into the corresponding cleavage site would inactivate the bla target gene, and therefore that E. coli undergoing such events would not be viable upon selection for $A m p^{R}$. Indeed, among the few $A m p^{R}$ colonies recovered following either CRISPR-control (green box) or Pro-AG (blue box) treatments (i.e., induction of Cas $9+\lambda$ Red), sequence analysis showed that $100 \%(30 / 30)$ carried unaltered pET plasmids with intact bla gene-coding sequences at the gRNA target site (Fig. 1d, bottom panel). These rare examples of $A m p^{R}$ E. coli colonies that evaded CRISPR or Pro-AG editing, likely from incomplete target cleavage of the high copy number plasmid, are referred to hereafter as "escapers".

Pro-AG results in precise homology-mediated editing. In analogy to HDR-dependent copying of gene-drive elements in diploid organisms, enhanced gene targeting activity of Pro-AG over CRISPR-control (Fig. 1d) might reflect homology-mediated insertion of the gRNA cassette into the bla gRNA2/Cas9 cleavage site. We tested this hypothesis by incorporating a second selectable antibiotic resistance gene (gentamicin $-\mathrm{Gm}^{\mathrm{R}}$ ) into the target pET plasmid (pETg; Supplementary Table 1), allowing recovery of bla gene-edited plasmids by selection on $\mathrm{Gm}$ plates (Supplementary Fig. 1). Consistent with high efficiency insertional copying, virtually the entire decrement of CFU seen in Pro-AG vs. CRISPR-control systems under Cas $9+\lambda$ Red induction and $A \mathrm{mp}^{\mathrm{R}}$ selection could be recovered under $\mathrm{Gm}^{\mathrm{R}}$ selection (Fig. 2a, blue box). Likewise, as expected, all Pro-AG recovered $\mathrm{Gm}^{\mathrm{R}}$ colonies failed to grow on Amp plates (Fig. 2b, bottom panel). In contrast to the nearly full restoration of the $\lambda$ Red-induced CFU decrement observed upon $\mathrm{Gm}^{\mathrm{R}}$ selection with the Pro-AG regimen, no CFU recovery was observed under $\mathrm{Gm}^{\mathrm{R}}$ vs. Amp $\mathrm{R}^{\mathrm{R}}$ selection using the CRISPR-control protocol for targeting bla (Fig. 2a, green box). 
a

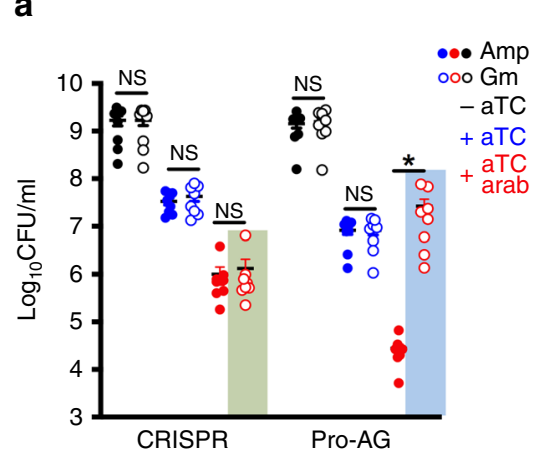

b

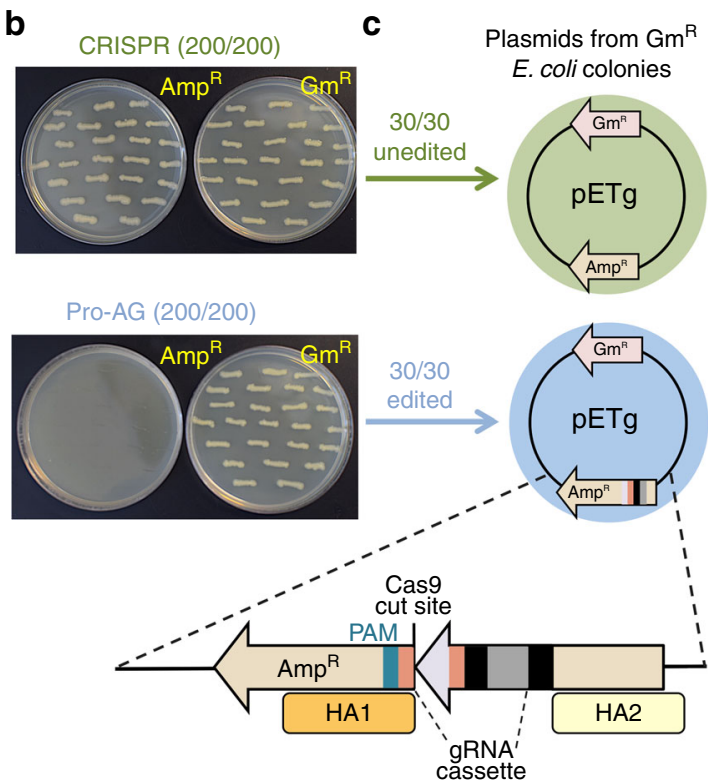

Fig. 2 Efficient reduction of Amp ${ }^{R}$ CFU by Pro-AG results from homology-mediated insertion of active gRNA cassettes into the bla target gene. a Selection for E. coli CFU on ampicillin (Amp, filled dots) or gentamicin (Gm, open dots) plates following CRISPR-control- or Pro-AG-mediated targeting of the dual antibiotic-resistant target plasmid pETg (see pETg plasmid schematic in c from single colonies grown in the presence ( + aTc, blue dots), in the absence ( - aTc, black dots) of anhydrotetracycline for induction of Cas9, or in combination of aTc and arabinose ( + aTc + arab, red dots) for induction of Cas 9 and $\lambda$ Red, respectively. $\mathbf{b}$ Individual colonies isolated from gentamicin plates following Cas 9 and $\lambda$ Red induction under CRISPR (green shaded box) or Pro-AG (blue shaded box) regimens in a were streaked on new ampicillin ( $A m p^{R}$, left images) and gentamicin ( $\mathrm{Gm}^{\mathrm{R}}$, right images) plates. Representative images of 200 colonies struck from CRISPR-control (top) or Pro-AG (bottom) are shown. c DNA sequence analysis of plasmids isolated from single colonies in $\mathbf{b}$ recovered from either the CRISPR-control regimen ( $A m p^{R}$ or $\mathrm{Gm}^{\mathrm{R}}$ plates; green arrow) or the Pro- $A \mathrm{G}$ regimen ( $\mathrm{Gm}{ }^{\mathrm{R}}$ plates; blue arrow). All 30 CRISPR-derivative clones analyzed revealed a fully intact pETg plasmid (unedited, green circle), whereas all 30 Pro-AG-derivative clones analyzed carried a perfect insertion of the homology-flanked gRNA2 expression cassette into the bla gene (zoom-in bottom scheme). The gRNA expression cassette is composed of the gRNA scaffold (purple), 20 bp gRNA-targeting sequences (pink and black), and the constitutive tet promoter (gray). Also indicated are the Cas9 cleavage site, and homology arms (HA1, dark yellow box and HA2, light yellow box) that flank the gRNA2 cleavage site in the bla target gene carried on the $\mathrm{pETg}$ plasmid. Data in a are plotted as the mean \pm SEM, representing three independent experiments performed in triplicate and analyzed by Student's $t$ test. N.S. $=$ not significant $(P>0.05) \star P<0.05$. Source Data are available in the Source Data file.

A clear prediction of the Pro-AG hypothesis examined above is that all recovered $\mathrm{Gm}^{\mathrm{R}}$ colonies should carry a precise insertion of the gRNA expression cassette. Indeed, analysis of $30 \mathrm{Gm}$-selected pETg plasmids from Pro-AG single colonies confirmed that the DNA cassette carried between the two homology arms, including the full gRNA2 scaffold and its promoter (Fig. 2c, bottom scheme), were perfectly copied from the donor plasmid (pProAGAmp, Fig. 1c; Supplementary Table 1) into the gRNA2 cleavage site of the bla target gene in all clones (Fig. 2c, blue circle). Thus the function of the targeted bla gene is disrupted by insertion of the gRNA expression cassette within its protein coding region. In contrast, all CRISPR-control-treated unedited E. coli escaper colonies recovered on $\mathrm{Gm}^{\mathrm{R}}$ selection regrew under selection for $A m p^{R}$ (Fig. 2b, top panel). Consistent with such escapers having evaded CRISPR-mediated mutagenesis, all (30/30) examined $A m p^{R}+G m^{R}$ CRISPR escapers displayed intact (unedited) bla gRNA2 target sites (Fig. $2 c$, green circle). In aggregate, the above findings support the hypothesis that the greatly enhanced reduction of $A m p^{R}$ CFU observed upon Pro-AG versus CRISPR-control editing configurations is quantitatively attributable to homologymediated insertion of gRNA2 sequences into bla-coding sequences on the target pETg plasmid. Of note, each insertional editing event expands the pool of functional gRNA donor scaffolds with extended homology arms in their newly copied plasmid context, which may initiate a chain reaction accelerating further insertional events.

As mentioned above, E. coli "escaper" colonies that evaded ProAG editing showed intact target sequences (Fig. 1d, bottom panel).
We hypothesized that inefficient Cas9 cleavage in escaper cells could reflect competition with the bacterial DNA repair system. We tested this idea by comparing Pro-AG editing of the bla gene target carried by the pETg plasmid in WT vs. $\triangle r e c A E$. coli (Supplementary Fig. 1b). A significant reduction of $\mathrm{Amp}^{\mathrm{R}}$ CFUs was obtained in $\triangle r e c A$ cells, under Cas9 induction alone or in combination with $\lambda$ Red (Supplementary Fig. 5a). All target plasmids analyzed from both $\mathrm{Gm}^{\mathrm{R}} \mathrm{WT}$ and $\triangle$ recA E. coli colonies bore precise gRNA2 insertions into bla target gene (Supplementary Fig. 5b), paralleling the editing efficiencies observed in previous experiments (Fig. 2c). Although these findings indicate that RecA is not required for copying the gRNA2 expression cassette from donor plasmid to target gene, they also suggest that Pro-AG escaper cells are partially protected in a RecA-dependent manner. An additional previously observed mechanism of escape from CRISPR-Cas9-mediated editing of $E$. coli involves mutations in the gRNA-harboring plasmid ${ }^{8,10}$. Consistent with similar mechanisms operating in CRISPR-based editing experiments, analysis of the pPro-AG (Amp) plasmid from escaper cells revealed that 50\% of gRNA launching plasmids recovered from escaper lines harbored deletions spanning the operator region, the gRNA and/or the gRNA scaffold sequences (Supplementary Fig. 6a).

Pro-AG depends on homology sequences flanking the gRNA. The above-described Pro-AG configuration, wherein the gRNA expression cassette is flanked directly by homology arms, represents the minimal possible self-copying element. We wondered if 

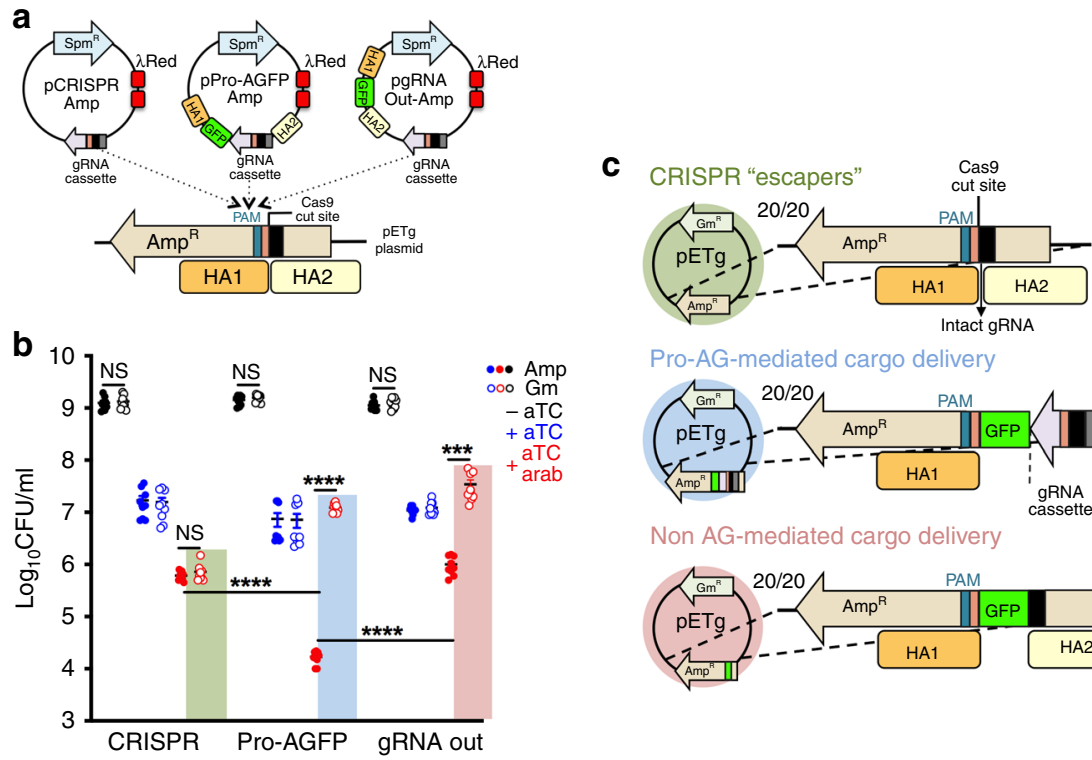

Pro-AG-mediated cargo delivery

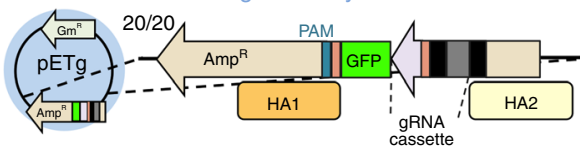

Non AG-mediated cargo delivery

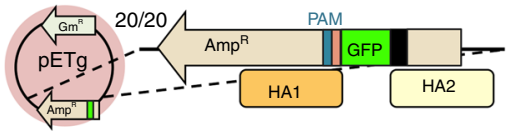

Fig. 3 The Pro-AG system mediates efficient editing and cargo delivery dependent on precise flanking of the gRNA cassette by homology arms of the target gene. a Schematic of plasmids used to compare performance of CRISPR-control (pCRISPR Amp), Pro-AGFP (pPro-AGFPAmp: gRNA2 + GFP within homology arms), and external placement of gRNA2 outside of the homology arms flanked cassette (pgRNA Out-Amp: GFP-only within homology arms, gRNA outside of HA-cassette). b Recovery of E. coli CFU following CRISPR-control or Pro-AG using the three different plasmid configurations indicated in a and the various induction conditions indicated in the key. c Sequence analysis of targeted plasmids. pETg plasmids recovered from CRISPR-control-treated colonies (green circle) all display intact target sequence ("escapers"). Analysis of all Pro-AGFP-recovered pETg plasmids (blue circle) confirmed precise insertion of the gRNA + GFP cassette at the gRNA cut site, which consists of the full gRNA cassette (scaffold, purple; gRNA, pink and black, and tet promoter, gray) plus GFP. Although the gRNAOut-targeted pETg configuration (red circle) resulted in 100-fold less efficient targeting of the bla target gene than the Pro-AGFP configuration, all plasmids isolated from colonies selected on Gm plates carried precise insertions of the GFP-only cassette. Data in $\mathbf{b}$ are plotted as the mean \pm SEM, representing three independent experiments performed in triplicate and analyzed by Student's $t$ test. N.S. $=$ not significant $(P>0.05) ;{ }^{\star \star \star} P<0.001 ;{ }^{\star \star \star \star} P<0.0001$. Source Data are available in the Source Data file.

this system might also be exploited to deliver additional DNA cargo sequences efficiently. As a test case, a green fluorescence protein (GFP) transgene was included as cargo with the gRNA2 expression cassette between the bla gene homology arms (pProAGFPAmp, Fig. 3a, Supplementary Table 1). Using the same experimental design described above for targeting $A m p^{R}+\mathrm{Gm}^{\mathrm{R}}$ plasmid pETg with the "gRNA-only" pPro-AG plasmid (Fig. 3a), the cargo-laden pPro-AGFP construct performed similarly to its minimal counterpart in reducing $A m p^{R} C F U$ versus the CRISPRcontrol regimen (Fig. 3b). As for the minimal Pro-AG element, regrowth of single Pro-AGFP colonies recovered on Gm plates following Cas $9+\lambda$ Red induction (Fig. 3b, blue box) revealed perfect insertion of the composite gRNA2:GFP cassette into the bla gRNA2 cleavage site in $100 \%$ of clones analyzed (Fig. 3c, blue circle). Again, escaper colonies recovered under the CRISPRcontrol regimen on $\mathrm{Gm}$ plates with Cas $9+\lambda$ Red induction (Fig. 3b, green box) maintained pristine unedited gRNA target sequences and also displayed an $A m p^{R}$ phenotype (Fig. 3c, green circle). We conclude that the Pro-AG system leads to highly efficient and precise insertion of the gRNA2:GFP cargo bearing cassette into the gRNA2 bla gene target site.

A defining feature of self-amplifying CRISPR-based gene-drive systems in diploid organisms is insertion of the gRNA-bearing cassette at the exact genomic site where the gRNA directs target cleavage $^{17}$. If amplification of the gRNA gene dosage resulting from its being actively copied contributed to the enhanced efficiency of the Pro-AG system, then placement of the gRNA outside of the homology arm cassette (pgRNAOut-Amp) (Fig. 3a, Supplementary Table 1) would significantly reduce editing efficiency of the target plasmid. Indeed, the gRNA-Out configuration performed comparably to the CRIPSR-control protocol, leading only to an $\sim 3 \log _{10}$-fold reduction in $\mathrm{CFU}$ under $\mathrm{Amp}^{\mathrm{R}}$ selection and Cas $9+\lambda$ Red + induction (Fig. $3 b$ ). Thus, the entire boost in $\mathrm{Amp}^{\mathrm{R}} \mathrm{CFU}$ reduction provided by the Pro-AG system was abrogated by placing the gRNA outside of the homology arms, consistent with the hypothesis that the Pro-AG process acts via a positive feedback amplification mechanism (Fig. 3b). Although the gRNA-out configuration eliminated the $\sim 2$-log increment in CFU reduction observed with Pro-AGFP arrangement under $A m p^{R}$ selection, significant $C F U$ recovery was nonetheless observed upon $\mathrm{Gm}^{\mathrm{R}}$ selection, suggesting that a large fraction of the baseline DNA breaks induced by CRISPRcontrol conditions were now being repaired by precise copying of the homology-flanked GFP-only cassette. Indeed, all 20 plasmids analyzed from $\mathrm{Gm}^{\mathrm{R}}$ colonies under the gRNA-Out regimen (Fig. 3b, red bar) carried perfect insertions of the GFP-only cassette within the gRNA2 cleavage site of bla (Fig. 3c, red circle).

Amplification of the gRNA contributes to Pro-AG performance. As copying of the gRNA2 expression cassette from a low (pPro-AG) to a high copy number plasmid (pETg) significantly amplified gRNA gene number and hence expression levels, we wondered whether the enhanced performance of Pro-AG versus CRISPR-control could be wholly attributable to this effect. We addressed this question by comparing $A m p^{R}$ CFU reduction using CRISPR-control versus Pro-AG configurations in a situation where the bla-targeting gRNA2 was encoded from the outset on a high copy number plasmid, namely the pETg target plasmid itself, which would limit gRNA amplification to at most twofold. E. coli harboring pCas9 and p $\Delta$ gRNA (Supplementary Table 1) expressing Cas 9 and $\lambda$ Red, respectively, were transformed with 
either pETgCRISPR or pETgPro-AG plasmids (Supplementary Fig. 7a, Supplementary Table 1). Using our earlier CRISPRcontrol and Pro-AG-editing protocols under Amp selection (Supplementary Fig. 1), Pro-AG performed 1-logfold more efficiently than CRISPR-control in reducing Amp ${ }^{\mathrm{R}}$ CFUs (Supplementary Fig. 7b). Although this Pro-AG gain is less than that achieved when gRNA2 was launched from a low copy plasmid, where we observed 2-logfold improved efficiencies, this more modest enhancement suggests that the full Pro-AG effect is not solely attributable to copy number amplification of gRNA2 (Fig. 1d). As before, significant CFU recovery occurred on Gm plates following Pro-AG editing (Supplementary Fig. 7b, top panel, blue shading), with perfect insertions of gRNA cassette into bla-coding sequences in $100 \%$ of pETgPro-AG plasmids analyzed (Supplementary Fig. 7b, bottom panel). We conclude that amplification of the homology-flanked gRNA, which may replicate itself $\sim 50$ times in a high copy plasmid, contributes significantly to the enhanced performance of Pro-AG. Additional mechanisms such as increased homology sequence length and double-strand DNA break repair may also contribute to enhanced Pro-AG efficiency compared with CRISPR only controls.

Pro-AG acts via a self-amplifying mechanism. As yet another approach to assess the role of cassette amplification in the enhanced performance of Pro-AG versus CRISPR-control paradigms we made use of the temperature sensitive nature of replication of the low copy number gRNA2 donor plasmids. Switching overnight growth cultures from $30^{\circ} \mathrm{C}$ (permissive temperature for plasmid replication) to $37^{\circ} \mathrm{C}$ (non-permissive temperature) during the editing protocol reduced $\mathrm{Spm}^{\mathrm{R}} \mathrm{CFU}$ that harbor the gRNA2 plasmid by $\sim 2.5$-logfold (Supplementary Fig. $8 \mathrm{a}$ ). As in previous experiments, Amp selection produced a significant reduction of $\mathrm{Amp}^{\mathrm{R}}$ CFUs for both CRISPR-control and Pro-AG configurations following Cas 9 and $\lambda$ Red induction $(+a T c+$ arab) at $30^{\circ} \mathrm{C}$, which was more pronounced for Pro-AG. In contrast, only the Pro-AG regimen, and not CRISPR-controls, also reduced $A m p^{R}$ CFUs following Cas9 $+\lambda$ Red induction $(+\mathrm{aTc}+\mathrm{arab})$ at $37^{\circ} \mathrm{C}$ (Supplementary Fig. 8b). Based on these several independent lines of corroborating evidence, we conclude that incorporating the gRNA ( \pm cargo) between homology arms to generate the active genetic cassette greatly increases targeting efficiency of a high copy number plasmid via a self-sustaining positive feedback loop.

Pro-AG is amenable to manipulation of large plasmids. Large plasmids carried by various pathogens pose an important health problem and are challenging to manipulate by traditional methods. We wondered whether the enhanced Pro-AG system might again offer advantages in such a context. We chose an E. coli strain harboring the pCas9 plasmid and $\mathrm{a} \sim 50 \mathrm{~Kb}$ cosmid vector (Supercos SV305, Supplementary Table 1) carrying Amp and Km selection markers as a test case. As in previous experiments, cells were transformed with either the pCRISPR Amp control or pProAG (SuperCos) plasmids, the latter adopting a Pro-AG configuration to target the bla gene encoded on the cosmid (Supplementary Fig. 9a). Following our standard editing protocols (Supplementary Fig. 1), we observed a 1-logfold greater reduction in $\mathrm{Amp}^{\mathrm{R}} \mathrm{CFUs}$ with the Pro-AG regimen than CRISPR-control following Cas 9 and $\lambda$ Red induction $(+\mathrm{aTc}+\mathrm{arab})$ (Supplementary Fig. 9b, top panel). Indicative of Pro-AG-mediated double-strand DNA break repair, significant CFUs were rescued under $\mathrm{Km}$ selection (Supplementary Fig. 9b, top panel, blue shading box), and $100 \%$ of the Supercos SV305 cosmids isolated and sequenced showed a precise gRNA cassette insertion into bla (Supplementary Fig. 9b, bottom panel). Thus Pro-AG is well suited for gene editing large multi-copy plasmids, with efficient homology-mediated insertion of gRNA sequences into the targeted coding region.

CRISPR and Pro-AG are equivalent for single locus editing. Given its efficiency in meeting the challenging task of targeting a high and moderate copy number plasmids, we asked whether the Pro-AG approach could similarly be employed to insert gRNAonly or gRNA + GFP cargo cassettes into a single-copy chromosomal target (e.g., the lac $Z$ gene). We found that Pro-AG configurations indeed performed with high efficiency in this context as well (Supplementary Text and Supplementary Figs. 10 and 11). Because in this context there is no need for cassette amplification provided by Pro-AG, we predicted that targeting efficiency would not depend on the placement of the gRNA between homology arms. This expectation was confirmed by experiments in which the gRNA was placed between (gRNA-in) or outside (gRNA-out) of the GFP-containing cassette since both configurations performed equivalently in precise editing of the lacZ target gene (Supplementary Fig. 12).

\section{Discussion}

Cumulatively, our results indicate that the Pro-AG configuration is 2-3 orders of magnitude more efficient in disrupting the activity of a high copy number target gene (bla) than the CRISPRcontrol arrangement, yielding to a $4-5 \log _{10}$-fold reduction in $A m p^{R}$ E. coli, fully attributed to precise insertion of the gRNA ( \pm cargo) cassette from the editing vector into the targeted gRNA cleavage site. Increased potency of the Pro-AG versus CRISPRcontrol configuration in reducing antibiotic-resistant CFUs depends on the gRNA cassette being precisely flanked by bla homology arms, suggesting that an amplifying positive feedback loop occurs from increasing gRNA copy number, a potential ratelimiting component of the system. The high efficiencies of accurate Pro-AG cassette copying in bacteria are comparable to those attained by gene-drive systems in diploid organisms $(>90 \%)^{1-3,18}$. In addition to its high efficiency, Pro-AG maintains an ability to perform precise and potentially subtle edits of a target gene rather than simply eliminating the target sequence or bacteria carrying that locus.

Multiple genome-engineering applications could ultimately benefit from incorporation of the Pro-AG platform, including elimination of bacterial virulence factors carried by diverse episomal elements and resistance determinants in commensal bacteria as well as different prokaryotic pathogens, scrubbing antibiotic resistance genes from bacteria in the environment ${ }^{19}$, livestock, inland fish farms, or sewage treatment ponds ${ }^{20}$, reprogramming genetic circuits impacting bacterial physiology, or tailoring interactions among the microbiota in environmental or host niches. For each of these applications suitable delivery systems such as phage vectors ${ }^{21}$ or conjugative plasmids ${ }^{22}$ would need to be developed and tailored to the specific contexts in which they were being deployed.

\section{Methods}

Strains and culture conditions. E. coli strain MG1655 WT and $\Delta$ RecA were provided by the B. Palsson and Susan Lovett Laboratories, respectively. Liquid cultures of $E$. coli were grown in LB medium. When appropriate, antibiotics were added as following: chloramphenicol (Cm, $25 \mu \mathrm{g} / \mathrm{ml}$ ), ampicillin (Amp, $100 \mu \mathrm{g} / \mathrm{ml}$ ), spectinomycin $(\mathrm{Spm}, 50 \mu \mathrm{g} / \mathrm{ml})$, and gentamicin $(\mathrm{Gm}, 10 \mu \mathrm{g} / \mathrm{ml})$.

Plasmid construction. All constructs used in this study are listed in Supplementary Table 1 with primer sequences provided in Supplementary Table 2. Plasmids pKDsgRNA (pCRISPR) and pCas9-CR4 (pCas9) (Table S1) were purchased from Addgene (Cambridge, MA). pCRISPR(AmpgRNA1), pCRISPR (AmpgRNA2) and pCRISPRlacZ were built as previously described ${ }^{9}$. The $20 \mathrm{bp}$ targeting sequences of the gRNAs were cloned into pKDsgRNA using circular 
polymerase extension cloning (CPEC) ${ }^{23}$ of two linear PCR fragments (F1, $3 \mathrm{~kb} ; \mathrm{F} 2$, $4 \mathrm{~kb}$ ). For pCRISPR(AmpgRNA1) (Supplementary Table 1), F1 and F2 were obtained using the paired primers 13_FwAmpgRNA1/14_RvF1 and 15_FwF2/ 16_RvAmpgRNA1 (Supplementary Table 2), respectively. For pCRISPR (AmpgRNA2) (Supplementary Table 1), F1 and 1 and 2 were obtained using the paired primers 17_FwAmpgRNA2 /14_RvF1 and 18_RvAmpgRNA2 /15_FwF2 (Supplementary Table 2), respectively. For pCRISPRlacZ (Supplementary Table 1), F1 and F2 were obtained using the paired primers 33_FwlacZgRNA/ 14_RvF1 and 34_RvlacZgRNA/15_FwF2 (Supplementary Table 2), respectively. PCR products with $\sim 280 \mathrm{bp}$ of overlapping homology sequences and $20 \mathrm{bp}$ of overlap in the protospacer region were DpnI digested for at least $15 \mathrm{~min}$ then gelpurified. Fragments 1 and 2 were mixed together in equal amounts (200 ng each) and CPEC cloned with 15 cycles and Phusion High-Fidelity Polymerase (NEB). In all, $2.5 \mu \mathrm{l}$ of the mixture was used to transform one-shot Stbl3 chemically competent $E$. coli cells (Thermo Fisher)

Plasmids expressing Pro-AG configurations pPro-AG(Amp), pPro-AG(lacZ), and pPro-AG Super-Cos (Supplementary Table 1) were built in two Gibson (NEBuilder, NEB) assembly steps using two linear PCR fragments with flanking overlapping sequences. For the pPro-AG(Amp) plasmid construct, the first step consisted of cloning bla sequence homology arm 1 in pCRISPR(AmpgRNA2) to generate the plasmid pCRISPR(AmpgRNA2 + HA1) (Supplementary Table 1) by amplification of two PCR fragments (F1 and F2) with paired primers 37_FwgRNA2/ 38_RvgRNA2 and 39_FwHA1Amp/40_RvHA1Amp (Supplementary Table 2), respectively. A second step consisted of cloning bla sequence homology arm 2 in pCRISPR(AmpgRNA2 + HA1) to generate plasmid pPro-AG(Amp)

(Supplementary Table 1) by amplification of two PCR fragments (F1 and F2) with paired primers 41_FwgAmpHA1/42_RvgAmpHA1 and 43_FwHA2/44_RvHA2 (Supplementary Table 2), respectively. For the pPro-AG(lacZ) plasmid construct, a first step consisted of cloning lac $Z$ sequence homology arm 1 in pCRISPR(lacZ) to generate pCRISPR(lac $Z+\mathrm{HA} 1$ ) plasmid (Supplementary Table 2 ) by amplification of two PCR fragments (F1 and F2) with paired primers 51_FwlacZgRNA/ 52_RvlacZgRNA and 53_FwHAllacZ/54_RvHAllacZ (Supplementary Table 2), respectively. A second step consisted of cloning lac $Z$ sequence homology arm 2 in pCRISPR(lacZ + HA1) to generate plasmid pPro-AG(lacZ) (Supplementary Table 1) by amplification of two PCR fragments (F1 and F2) with paired primers 55_FwlacZHA1/56_FwlacZHA1 and 57_FwlacZHA2/58_RvlacZHA2 (Supplementary Table 2), respectively. For the pPro-AG Super-Cos plasmid construct, we first cloned bla sequence homology arm 1 in pCRISPR (AmpgRNA2) to generate the plasmid pCRISPR(AmpgRNA2 + SV3B05HA1HA1) (Supplementary Table 1) by amplification of two PCR fragments (F1 and F2) with paired primers 106_FwHA1Supercos/107 RvHA1Supercos and 108_FwpKDsAmp2/109_RvpKDsAmp2 (Supplementary Table 2), respectively. A second step consisted of cloning bla sequence homology arm 2 in pCRISPR (AmpgRNA2 + SV3B05HA1HA1) to generate plasmid pPro-AG Super-Cos (Supplementary Table 1) by amplification of two PCR fragments (F1 and F2) with paired primers 110_Fw HA2Supercos/111_Rev HA2Supercos and Pr_112Fw CosHA1/Pr_113Rv CosHA1 (Supplementary Table 2), respectively.

Plasmids pETg, pPro-AGFP(Amp), pPro-AGFP(lacZ), pETgCRISPR, and pETgPro-AG (Supplementary Table 1) were generated by amplification of two linear PCR fragments (F1 and F2), with flanking overlapping sequences for Gibson assembly (NEBuilder, NEB). Paired primers 59_FwpET/60_RvpET and 61_FwGm/ 62_RvGm (Supplementary Table 2) were used to amplify PCR F1 and F2 to assemble pETg. Paired primers 69_FwP-AG(Amp)/73_RvP-AG(Amp) and 72_FwGFP-Amp/68_RvGFP-Amp (Supplementary Table 2) were used to amplify PCR F1 and F2 to assemble pPRO-AGFP(Amp). Paired primers 63_FwP-AG(lacZ)/ 64_RvP-AG(lacZ) and 65_FwGFP-lacZ/66_RvGFP-lacZ (Supplementary Table 2) were used to amplify PCR F1 and F2 to assemble pPro-AGFP(lacZ). Paired primers 98_FwProAG/99-RvPro-AG and 100_FwpETg(Pro-AG)/101_RvpETg(Pro-AG) were used to amplify PCR F1 and F2 to assemble pETgCRISPR. Paired primers 102_FwCRISPR/103-RvCRISPR and 104_FwpETg(CRISPR)/105_RvpETg(CRISPR) were used to amplify PCR F1 and F2 to assemble pETgCRISPR.

For the pgRNAout (Amp) and pgRNAout (lacZ) plasmid constructs, pCRISPR (AmpgRNA2) and pCRISPR(lacZ), respectively were linearized with the NcoI restriction enzyme. Subsequently, three fragments with flanking overlapping sequences (F1, F2, and F3) were PCR amplified as follows. For pgRNAout (Amp) construct, homology arms 1 (F1) and 2 (F2) from pPro-AG(Amp) and $g f p$ (F3) from plasmid \#48138 (Addgene), using primer pairs 67_FwHA1-GFPout/ 68_RVHA1-GFPout, 69_FwHA2-GFPout/70_RvHA2-GFPout and 71_FwGFPGFPout/72_RvGFP-GFPout (Supplementary Table 2), respectively. For pgRNAout (lacZ) construct, homology arms 1 (F1) and 2 (F2) from pPro-AG(lacZ) and $g f p$ (F3) from plasmid \#48138 (Addgene), using primer pairs 88_FwHA1-lacZout/ 89 RvHA1-lacZout, 92 FwHA2-lacZout/93 RvHA2-lacZout and 90 FwGFPlacZout/91_RvGFP-lacZout (Supplementary Table 2), respectively. Gibson assembly was carried out with the linearized vectors and the three corresponding overlapping PCR fragments to generate pgRNAout(Amp) and pgRNAout(lacZ) (Supplementary Table 1). All Gibson assembly reactions were transformed into NEB 5-alpha competent E. coli cells.

E. coli transformation. Competent cells were prepared as previously described (Short Protocols in Molecular Biology, Chapter 1). For all plasmid transformations,
$50 \mu \mathrm{l}$ of aliquoted cells were gently thawed on ice, followed by addition of plasmid DNA prepared by QIAprep Spin Miniprep Kit (Qiagen), with $20 \mathrm{ng}$ of each plasmid added to the transformation mix. E. coli cells were electroporated with the $1 \mathrm{~mm}$ Gene Pulser cuvette (Bio-Rad) at $1.6 \mathrm{kV}$ and immediately resuspended in $250 \mu \mathrm{l}$ super optimal broth with catabolite repression media. Cells were allowed to recover for $2 \mathrm{~h}$ at $30^{\circ} \mathrm{C}$ (for cells transformed with pKDsgRNA derived plasmids) and for $1 \mathrm{~h}$ at $37^{\circ} \mathrm{C}$ for cells transformed with pETg plasmid. Serial dilutions of cells were plated on LB with the corresponding antibiotic and they were incubated at $30^{\circ} \mathrm{C}(48 \mathrm{~h})$ or $37^{\circ} \mathrm{C}(24 \mathrm{~h})$, respectively.

Induction of Cas9 and Lambda-Red enzymes. Single E. coli colonies obtained on plates after transformation were resuspended in $60 \mu \mathrm{LB}$, which served as inoculum for $5 \mathrm{ml} \mathrm{LB}$ overnight cultures $(\sim 15 \mathrm{~h})$ grown at $30^{\circ} \mathrm{C}$ with shaking $(200 \mathrm{rpm})$. When appropriate, Cas 9 expression was induced in cells carrying the pCas 9 plasmid (Supplementary Table 1) by adding $100 \mathrm{ng} / \mathrm{ml}$ anhydrotetracycline (aTc, Abcam) to the broth media. Similarly, when desired, $\lambda$-Red expression was induced in cells carrying pKdsgRNA derivative plasmids (Supplementary Table 1) by adding $50 \mathrm{~mm}$ arabinose (arab, Sigma) to the broth media during editing steps (Supplementary Fig. 1).

E. coli colony counts. CFU were determined similarly to the miniaturized plating method described previously with small modifications. In brief, $25 \mu \mathrm{l}$ of overnight culture, and dilution series of $10^{-1}$ to $10^{-8}$, were spotted in triplicate on LB plates containing the appropriate antibiotic selection and incubated overnight at $30^{\circ} \mathrm{C}$. No notable differences were observed when arab or aTc were added to the plates for $\lambda$-Red or Cas 9 induction, respectively.

Sequence analysis of editing events. For bla-editing events, $\mathrm{pETg}$ plasmids and Super-Cos (SV3B06) cosmids were purified from $A m p^{S} / \mathrm{Gm}^{\mathrm{R}} \mathrm{Amp} / \mathrm{Sm}{ }^{\mathrm{R}}$, respectively, single edited E. coli colonies by QIAprep Spin Miniprep Kit (Qiagen) and sequenced (Genewiz) with primer 35_FwAmp(ext) (Supplementary Table 2). pET-derivative plasmids from single escapers were analyzed following the same parameters. For lacZ-editing events, white E. coli colonies where quantified among the total white + blue mixed population of colonies on LB plates containing $1 \mathrm{~mm}$ IPTG and $0.03 \%(\mathrm{v} / \mathrm{v})$ Bluo-Gal (Teknova). Single white colonies were grown overnight at $37^{\circ} \mathrm{C}$ and total genomic DNA isolated by DNeasy Blood and Tissue (Qiagen). PCR products were obtained with Q5 DNA polymerase (NEB) and using primer pairs 49_FwlacZseq and 50_RvlacZseq, followed by sequencing analysis (Genewiz) with primer 63_RvlacZint (Supplementary Table 2). Escaper blue colonies were also analyzed following the same parameters. gRNA plasmid constructs from escapers were sequenced with primers 27_Fw pKDSgRNAseq and 28_Rv pKDSgRNAseq.

Visualization and quantification of GFP expression. In all, $10 \mu \mathrm{l}$ aliquots from overnight cultures of $E$. coli exposed to the Pro-AGFP configuration in LB + 1 mM IPTG and under Cas 9 and $\lambda$-Red induction were mounted onto slides with coverslips. GFP fluorescence was visualized using a Zeiss Axio Observer.D1 fluorescence microscope. For GFP fluorescence quantification, single white colonies from E. coli lacZ-editing plates were homogenized in $200 \mu \mathrm{l}$ PBS and subsequently transferred to a 96-well plate. Optical density at $600 \mathrm{~nm}$ and GFP fluorescence at $510_{\mathrm{nm}}$ were measured by using an EnSpire Plate Reader (PerkinElmer)

Reporting summary. Further information on research design is available in the Nature Research Reporting Summary linked to this article.

\section{Data availability}

All data generated and analyzed during this study are included in the published article or provided in the Supplementary Information and are available from the corresponding author upon request. Source Data for Figs. 1-3 and Supplementary Figures 2-5, 7-12 are available in the Source Data file.

Received: 22 April 2019; Accepted: 15 November 2019; Published online: 16 December 2019

\section{References}

1. DiCarlo, J. E., Chavez, A., Dietz, S. L., Esvelt, K. M. \& Church, G. M. Safeguarding CRISPR-Cas9 gene drives in yeast. Nat. Biotechnol. 33, 1250-1255 (2015).

2. Gantz, V. M. \& Bier, E. The mutagenic chain reaction: a method for converting heterozygous to homozygous mutations. Science 348, 442-444 (2015).

3. Gantz, V. M. et al. Highly efficient Cas9-mediated gene drive for population modification of the malaria vector mosquito Anopheles stephensi. Proc. Natl Acad. Sci. USA 112, E6736-E6743 (2015). 
4. Hammond, A. et al. A CRISPR-Cas9 gene drive system targeting female reproduction in the malaria mosquito vector Anopheles gambiae. Nat. Biotechnol. 34, 78-83 (2016).

5. Grunwald, H. A. et al. Super-Mendelian inheritance mediated by CRISPRCas9 in the female mouse germline. Nature 566, 105-109 (2019).

6. Gratz, S. J. et al. Highly specific and efficient CRISPR/Cas9-catalyzed homology-directed repair in Drosophila. Genetics 196, 961-971 (2014).

7. Javed, M. R. et al. CRISPR-Cas system: history and prospects as a genome editing tool in microorganisms. Curr. Microbiol. 75, 1675-1683 (2018).

8. Jiang, W., Bikard, D., Cox, D., Zhang, F. \& Marraffini, L. A. RNA-guided editing of bacterial genomes using CRISPR-Cas systems. Nat. Biotechnol. 31, 233-239 (2013).

9. Reisch, C. R. \& Prather, K. L. J. Scarless Cas9-assisted recombineering (noSCAR) in Escherichia coli, an easy-to-use system for genome editing. Curr. Protoc. Mol. Biol. 117, 3138 31-31 3820 (2017).

10. Citorik, R. J., Mimee, M. \& Lu, T. K. Sequence-specific antimicrobials using efficiently delivered RNA-guided nucleases. Nat. Biotechnol. 32, 1141-1145 (2014).

11. Johnson, T. J. \& Nolan, L. K. Pathogenomics of the virulence plasmids of Escherichia coli. Microbiol. Mol. Biol. Rev. 73, 750-774 (2009).

12. Partridge, S. R., Kwong, S. M., Firth, N. \& Jensen, S. O. Mobile genetic elements associated with antimicrobial resistance. Clin. Microbiol. Rev. 31, pii: e00088-17 (2018).

13. Bassalo, M. C. et al. Rapid and efficient one-step metabolic pathway integration in E. coli. ACS Synth. Biol. 5, 561-568 (2016)

14. Cui, L. \& Bikard, D. Consequences of Cas 9 cleavage in the chromosome of Escherichia coli. Nucleic Acids Res. 44, 4243-4251 (2016).

15. Murphy, K. C. Lambda recombination and recombineering. EcoSal Plus 7, https://doi.org/10.1128/ecosalplus.ESP-0011-2015 (2016).

16. Thomason, L. C., Sawitzke, J. A., Li, X., Costantino, N. \& Court, D. L. Recombineering: genetic engineering in bacteria using homologous recombination. Curr. Protoc. Mol. Biol. 106, 116 11-39 (2014).

17. Gantz, V. M. \& Bier, E. The dawn of active genetics. Bioessays 38, 50-63 (2016).

18. Kyrou, K. et al. A CRISPR-Cas9 gene drive targeting doublesex causes complete population suppression in caged Anopheles gambiae mosquitoes. Nat. Biotechnol. 36, 1062-1066 (2018).

19. Ye, M. et al. A review of bacteriophage therapy for pathogenic bacteria inactivation in the soil environment. Environ. Int. 129, 488-496 (2019)

20. Pazda, M., Kumirska, J., Stepnowski, P. \& Mulkiewicz, E. Antibiotic resistance genes identified in wastewater treatment plant systems - A review. Sci. Total Environ. 697, 134023 (2019)

21. El Haddad, L., Harb, C. P., Gebara, M. A., Stibich, M. A. \& Chemaly, R. F. A systematic and critical review of bacteriophage therapy against multidrugresistant ESKAPE organisms in hjumans. Clin. Infect. Dis. 69, 167-178 (2019).

22. Hamilton, T. A. et al. Efficient inter-species conjugative transfer of a CRISPR nuclease for targeted bacterial killing. Nat. Commun. 10, 4544 (2019).

23. Quan, J. \& Tian, J. Circular polymerase extension cloning. Methods Mol. Biol. 1116, 103-117 (2014).

\section{Acknowledgements}

We thank Natalia Tchowri and Susan Lovett Laboratories for providing us the SuperCos (SV3B06) and E. coli $\triangle$ RecA strain, respectively. We also thank Donald Helinski for valuable advice in setting up the three-plasmid system as well as helpful comments on the manuscript. We also thank Eduardo Díaz, Marty Yanofsky, Sara Werner, and members of the Nizet and Bier labs for comments on the manuscript, Annabel Guichard, Justin Meyer, Suresh Subramani, and Sara Werner for early discussions to frame the Pro-AG strategy. These studies were supported by NIH grant R01 GM117321 and a Paul G. Allen Frontiers Group Distinguished Investigators Award to E.B., and a gift from the Tata Trusts in India to TIGS-UCSD and TIGS-India.

\section{Author contributions}

J.A.V. and S.S.K. performed the experiments and contributed to the experimental design and writing of the manuscript. V.N. and E.B. developed the concept for the project, contributed to the experimental design and writing of the manuscript.

\section{Competing interests}

E. Bier is a co-founder of the start-up companies Synbal and Agragene. All other authors declare no competing interests.

\section{Additional information}

Supplementary information is available for this paper at https://doi.org/10.1038/s41467019-13649-6.

Correspondence and requests for materials should be addressed to V.N. or E.B.

Peer review information Nature Communications thanks James DiCarlo and the other, anonymous, reviewer(s) for their contribution to the peer review of this work.

Reprints and permission information is available at http://www.nature.com/reprints

Publisher's note Springer Nature remains neutral with regard to jurisdictional claims in published maps and institutional affiliations.

(c) (i) Open Access This article is licensed under a Creative Commons BY Attribution 4.0 International License, which permits use, sharing, adaptation, distribution and reproduction in any medium or format, as long as you give appropriate credit to the original author(s) and the source, provide a link to the Creative Commons license, and indicate if changes were made. The images or other third party material in this article are included in the article's Creative Commons license, unless indicated otherwise in a credit line to the material. If material is not included in the article's Creative Commons license and your intended use is not permitted by statutory regulation or exceeds the permitted use, you will need to obtain permission directly from the copyright holder. To view a copy of this license, visit http://creativecommons.org/ licenses/by/4.0\%

(C) The Author(s) 2019 